\title{
Identification of tissular origin of particles based on autofluorescence multispectral image analysis at the macroscopic scale
}

\author{
Mathias Corcel $^{1,2}$, Marie-Françoise Devaux ${ }^{2}$, Fabienne Guillon $^{2}$ and Cécile Barron ${ }^{1 *}$ \\ ${ }^{1}$ UMR 1208 IATE, INRA-Supagro-UM-CIRAD, 2 Place P. Viala, 34000 Montpellier, France \\ ${ }^{2}$ UR 1268 BIA, INRA, Rue de la Géraudière, 44316 Nantes, France
}

\begin{abstract}
Powders produced from plant materials are heterogeneous in relation to native plant heterogeneity, and during grinding, dissociation often occurred at the tissue scale. The tissue composition of powdery samples could be modified through dry fractionation diagrams and impact their end-uses properties. If tissue identification is often made on native plant structure, this characterization is not straightforward in destructured samples such powders. Taking advantage of the autofluorescence properties of cell wall components, multispectral image acquisition is envisioned to identify the tissular origin of particles. Images were acquired on maize stem sections and ground tissues isolated from the same stem by hand dissection. The variability in fluorescence intensity profiles was analysed using principal component analysis. The correspondence between fluorescence profiles and the different tissues observed in maize sections was assessed based on histology or known compositional heterogeneity. Similar variability was encountered in fluorescence profiles extracted from powder leading to the potential ability to predict tissular origin based on this autofluorescence multispectral signal.
\end{abstract}

\section{Introduction}

Plants are heterogeneous materials made up of organs (stem, grains, leaves...) which are themselves made up of various tissues including different cell types. The composition, structure and relative proportions of the different tissues in the organ are under biological control and determine human end-uses. A grinding step is often a prerequisite to any plant processing and the dissociation of the organs during milling is often observed at the tissue scale. As an example, the cereal milling industry aims at separating wheat grains and recovering the starchy endosperm into flour whereas the most external tissues are collecting in bran fractions. The proportions of each tissue in the resulting fractions impact their end-use properties and the distribution of tissue within a single particle is required to understand, control and optimize the fractionation processes. Therefore the tissular origin of powders must be identified at the particle level. If the identification of tissues in structured organ is commonly performed through morphological information together with chemical staining, it remains challenging for powders. Tissue identification in powder is mainly based on their specific biochemical composition, which is theoretically the same after grinding. To observe tissue dissociation within particles, identification must be carried out at the micrometric scale and lead to envision spectroscopic analysis coupled to imaging. Considering the overall composition of plant tissues and their quite small differences in between, molecular spectroscopies such as Raman, infrared or UV-visible fluorescence are classically used to analyse plant tissues in microscopic sections [1,2]. However, to be applicable to plant particles analysis, the spectral imaging must deal with: low sample preparation (eg avoid sample section), a fine resolution $(<3 \mu \mathrm{m}$ per pixel), and at the same time, a large field of view to observe a large number of particles of variable size (from $50 \mu \mathrm{m}$ to $2 \mathrm{~mm}$ ). This implies a high speed of acquisition while keeping enough spectral information. Fluorescence macroimagers meet such requirements . ,In addition, many plant cell walls are known to be autofluorescent, with spectral signature specific to their phenolic composition. The objective of this work was to identify the tissue origin of plant particles based on their autofluorescence properties. Maize stem internode served as an example of plant material. It is made of two main regions: i) the inner one, the pith, which is constituted of vascular bundles (including both conducting tissues xylem and phloem surrounded by sclerenchyma cells) spread into parenchyma cells, and ii) the external one, the rind, which is rich in vascular bundles and sclerenchyma cells and surrounded by an epidermis on the most external part (Fig. 1) [3].

\footnotetext{
* Corresponding author: cecile.barron@supagro.inra.fr
} 


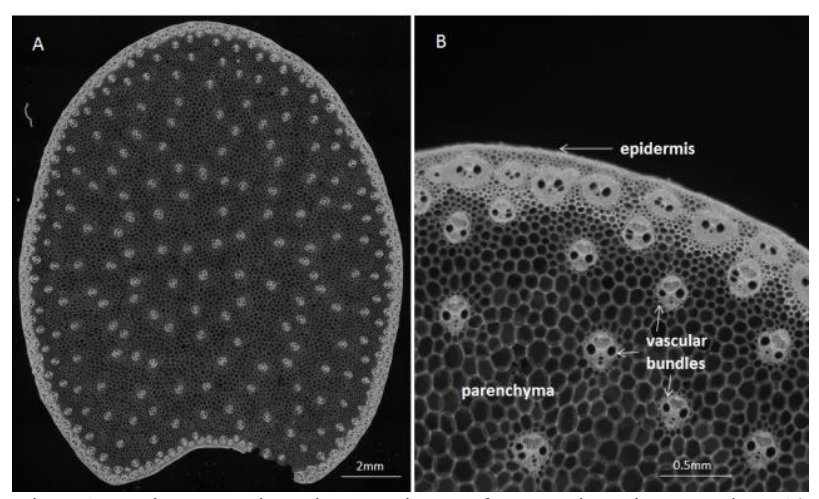

Fig. 1. Microscopic observation of a maize internode. A) Whole cross section issued of combined images B) one image with some maize tissues/structures underlined.

The method was developed on maize stem taken as a model plant, using a multispectral fluorescence imaging system. We propose to calibrate multivariate maps using data coming from the multispectral images of tissue sections and to validate the maps by projecting reference powders data issued from pure plant tissues.

\section{Material and Method}

\subsection{Material}

Cross sections and pure tissues were obtained from the same maize stem internode (Maxxis variety) in order to keep down the eventual compositional variability [4]. $150 \mu \mathrm{m}$ thick cross sections were cut with a vibratome (Microm, Microtech France) and stored in 70\% ethanol. Whole maize internode was also ground to obtain a powder including all the tissular variability (median particle size $154 \mu \mathrm{m}$, with values between $25 \mu \mathrm{m}$ and $700 \mu \mathrm{m})$. Pure tissues were obtained after meticulous hand dissection performed under a stereomicroscope. Considering the easiness of separation, parenchyma vascular bundle and epidermis were separated (Fig. 1), and then ground before image acquisition.

\subsection{Multispectral image acquisition}

A multi-zoom macroscope AZ100M (Nikon, Japan) has been equipped with 4 filter blocks to acquire images under UV, blue and green excitation light. Emission light was recovered through long pass filters and, taking advantage of the RGB channels of the colour camera (DSRiI, Nikon, Japan), each image were split in three channels and stack all together to obtain a 12 channels multispectral image (Fig. 2). The exposure times were fixed at U1 : $270 \mathrm{~ms}$, U2 : $522 \mathrm{~ms}$, Blue : $540 \mathrm{~ms}$ and Green : 990 ms. Channels names were: UA-B, UA-G, UA-R, UB-B, UB-G, UB-R, BL-B, BL-G, BL-R, GR-B, GR-G, and GR-R. The channels BL-B, GR-B and GR-G gave no autofluorescence signal considering the emission filters used but were kept to help to visualize each image in so-called multispectral images (Fig. 2).

The total magnification was set to $4 \mathrm{x}$ by combining the lens Plan Fluor 2x (NA: 0.2/WD: $45 \mathrm{~mm}$ ) and setting the optical zoom to 2 . The image size was $1280 \times 1024$ pixels, leading to a pixel size of $2.74 \mu \mathrm{m}$ and a field of view of $3.5 \times 2.8 \mathrm{~mm}^{2}$. In order to image the whole internode cross section (Fig. 1A), mosaic image were obtained by acquiring multiple images across the section using automated mode and an overlapping of $15 \%$ and stitching of these images into a single large image.

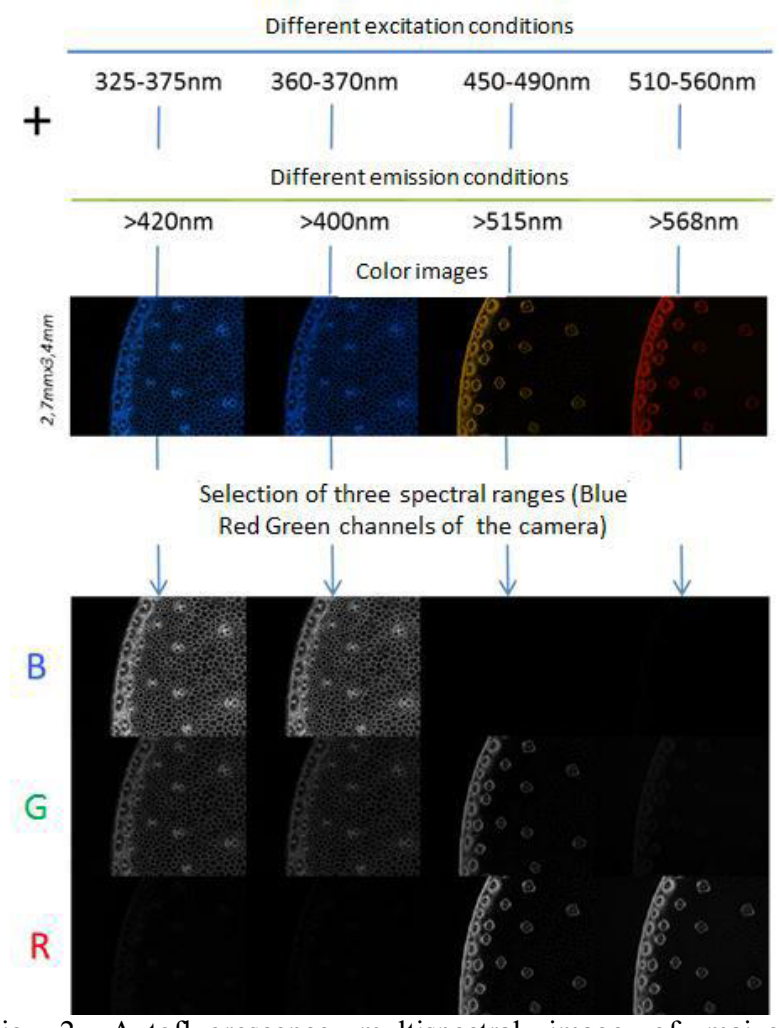

Fig. 2. Autofluorescence multispectral image of maize internode. Colour images and RGB channels.

\subsection{Data treatment}

Some pixels profiles from epidermis, parenchyma and sclerenchyma around vascular bundle were extracted using an interactive homemade function developed using the Matlab 2014a and the Image analysis toolbox (Mathworks, USA).

Principal component analysis was carried out, using Matlab 2014a., on the whole image section after normalisation and elimination of pixels out of the samples or saturated. Profiles of pixels acquired on powders were further projected on this principal component analysis.

\section{Results}

\subsection{Multispectral images of maize stem sections and particles.}

Autofluorescence multispectral images were acquired both on maize stem sections and maize powders (Figure 3). To enable comparison, exposure time ratios were set identical for all samples. The colour image is a representation of the 12 channels, obtained by averaging the four individual colour images obtained in each excitation/emission conditions. 

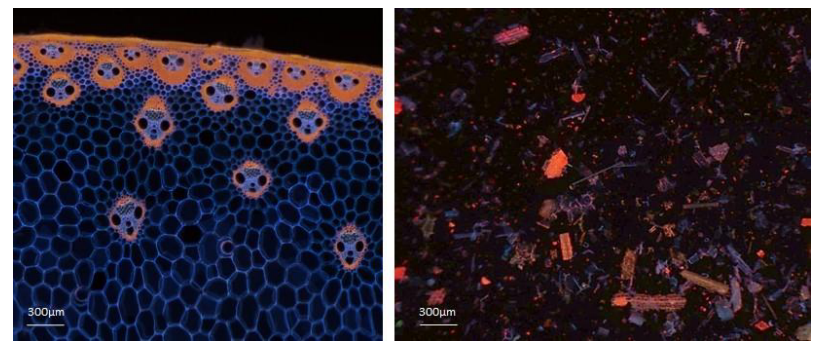

Fig. 3. Autofluorescence multispectral images of maize stem internode. A) cross section B) powder.

Maize stem structure could be easily differentiated in images of maize sections through their morphology (Fig.1) but also by their colour in the average representation of the multispectral image: parenchyma cell walls displayed a blue colour whereas epidermis or the sclerenchyma cells around vascular bundles were orange (Fig. 3A). Similar colour palette was observed in ground maize stem, with particles either orange or blue (Fig. 3B), reflecting the whole heterogeneity observed in a maize internode.

\subsection{Fluorescence profiles encountered in maize section}

In such multispectral images, each pixel is characterized by an intensity profile that can be considered as a spectral signature (Fig. 4). observed in the averaged multispectral image (Fig 3A). The presence of phenolic compounds such as hydroxycinammic acid (ferulic or para-coumaric acid) could explain this strong blue fluorescence under UV excitation [5]. Profiles extracted from epidermis were different, with low fluorescence intensity recovered under UV excitation but intense under blue or green excitation. Similar profile was observed for sclerenchyma cells but with higher fluorescence intensity emitted under UV excitation. This could be related to the orange colour observed in multispectral image and to the high lignin content encountered in sclerenchyma tissue [6].

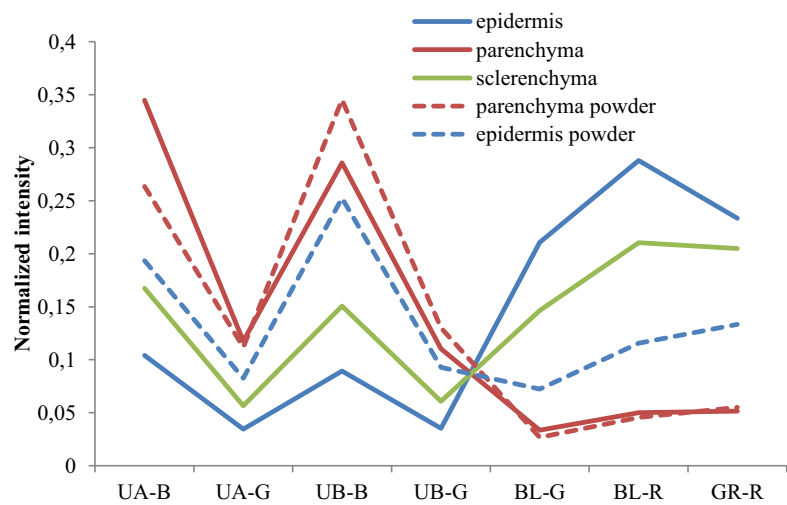

Fig. 4. Autofluorescence profiles of selected maize stem tissues either in cross-section or in powder.

Parenchyma showed intense fluorescence under UV excitation that could be related to the blue color
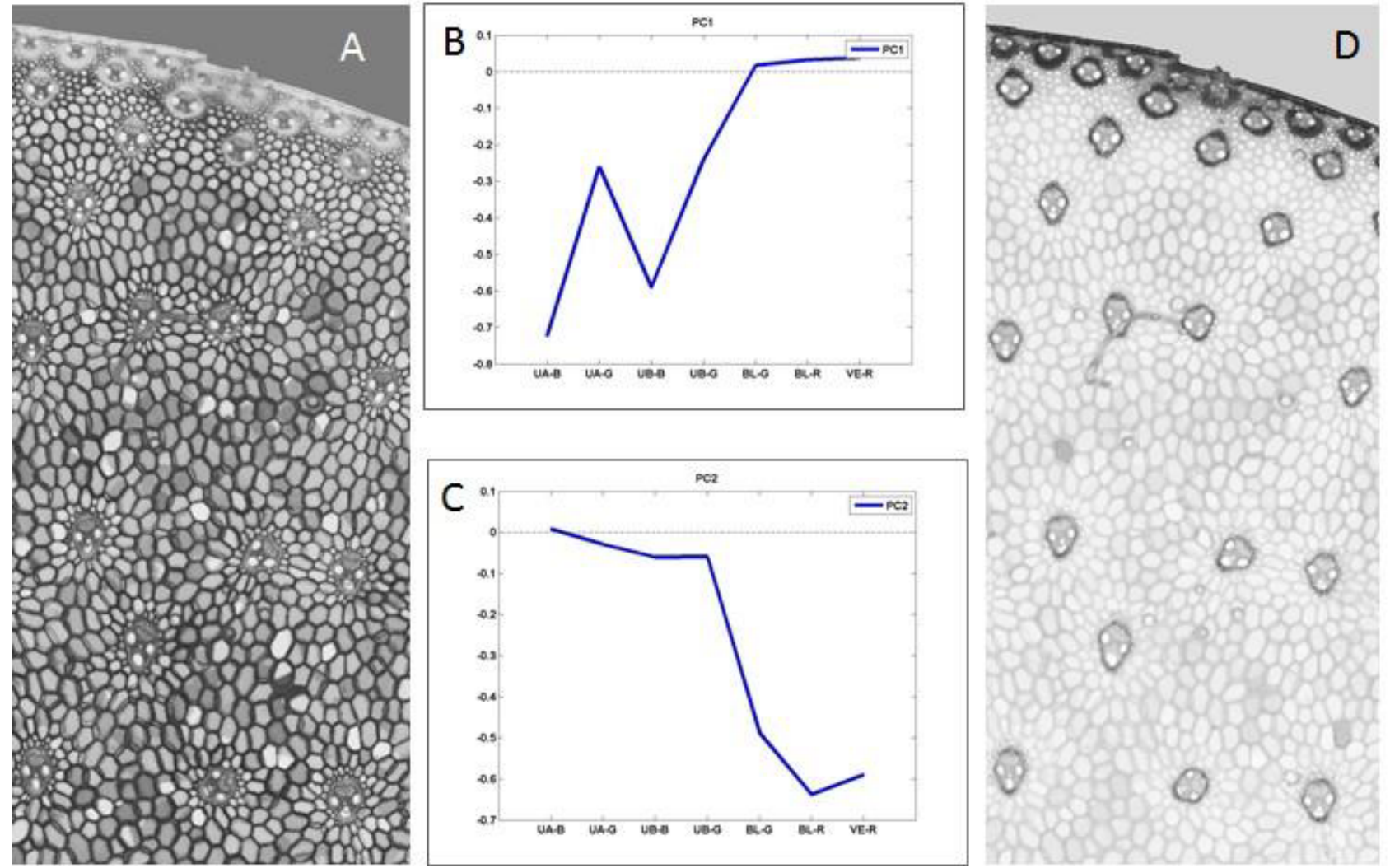

Fig. 5. Principal component analysis applied to autofluorescence images of maize stem cross section. A) Image of component 1, B) First loading, C) Second loading, D) Image component 2. 
Principal component analysis was applied to the whole pixels from the maize stem cross section to analyze their similarities and taking into account the whole variability. The variance explained by the two first components was $67 \%$ and 29\% respectively. The first loading (Fig. 5B) showed the autofluorescence intensities in UV filters in negative. In the component 1 image (Fig. 5A) a strong spatial heterogeneity is observed according to component 1 scores. The parenchyma pixels are observed in black indicating high fluorescence intensity under UV (Fig 5B) as observed in a typical fluorescence profile (Fig 4). On the contrary epidermis pixels were white indicating low fluorescence in these conditions, also in agreement with mean fluorescence profiles (Fig.4). Sclerenchyma pixels were in between, differently according to their location either in the pith or in the rind. The second loading showed the autofluorescence intensity in visible excitation (Fig. 5C). In the component 2 image (Fig. 5D) the parenchyma pixels are white indicating low fluorescence intensity under blue or green excitation, whereas epidermis or sclerenchyma pixels were black indicating strong fluorescence in these conditions.

\subsection{Fluorescence profiles variability encountered in maize powders compared to maize section}

Reference powders from parenchyma or epidermis were imaged with the same conditions as those applied to image internode sections. Pixels were extracted from pure tissue particles and their fluorescence profiles projected in the principal component analysis obtained from section (Fig. 6). Pixels of both tissues were largely found as expected from those obtained for tissues encountered in maize section. Parenchyma pixels showed slightly higher scores for component 2 that can be related to the overall higher fluorescence intensity observed under UV excitation in the fluorescence profiles (Fig. 4). Epidermis cells were found also with lower component 1 scores and higher component 2 scores. These both tissues projected in PC1/PC2 similarity map showed therefore a lower difference than the one expected from section analysis. This was in accordance with the average fluorescence profile in Fig. 4. If the structural state (powder vs section) was shown to have no impact on tissues extracted from wheat grain [7], this must be further investigated on maize stem in order to understand this discrepancy. The impact of particle size and thickness on fluorescence recovery should be integrated in the data exploration.

\section{Conclusions}

Autofluorescence multispectral imaging can be carried out either on plant sections or plant powders. The assignment of fluorescence signal was carried out specifically on maize stem sections based on histology or known compositional heterogeneity. Such methodology allows differentiating plant tissues according to their autofluorescence properties.

The variability in fluorescence signatures encountered in maize stem powder was similar to the variability observed in maize stem sections. According to the plant tissue, the spectral signature were kept or slightly modified. Confusions between plant tissues in powder seem higher than in plant section. This must be further investigated considering the specificity of each tissue and their powder characteristics.
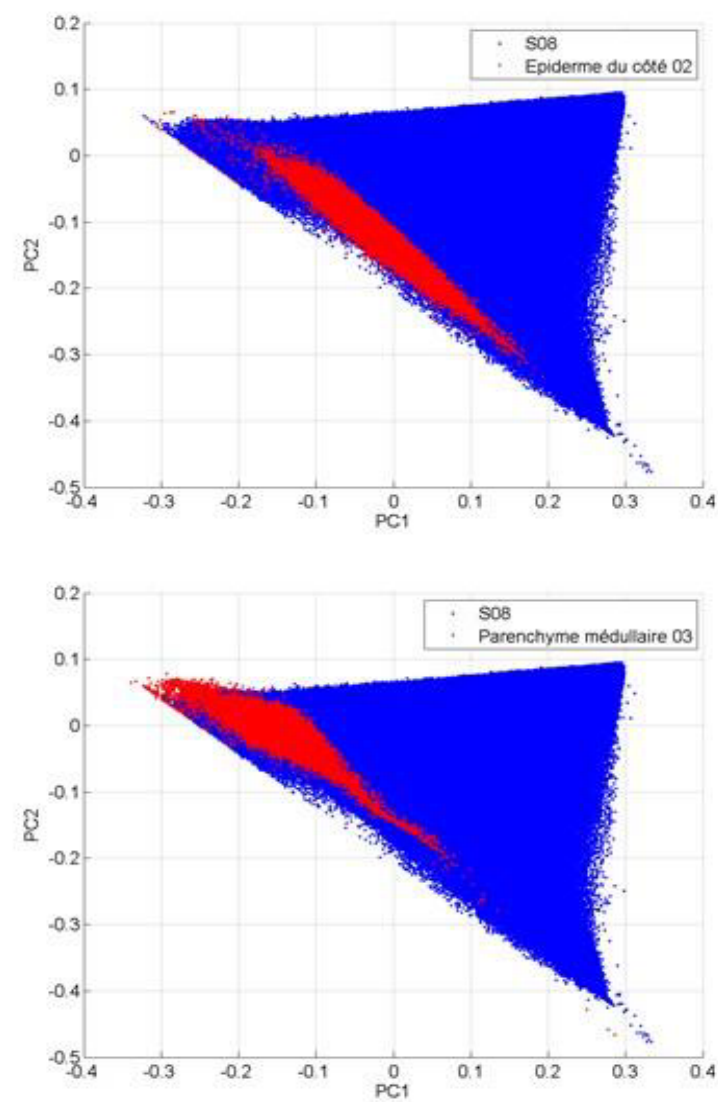

Fig. 6. Pixels from powder (in red) projected on principal component axis obtained from analysis carried out on pixels from maize section (in blue). A) Epidermis particles, B) Parenchyma particles

We would like to thank Aurélie Putois and Amélie Breysse for their help in sample preparation and image acquisition.

\section{References}

1. D.E. Akin, N. Ames-Gottfred, R.D. Hartley, R.G. Fulcher, L.L. Rigsby, 1990. Crop Sci., 30, 396-401, (1990)

2. L. Sun, BA Simmons, S. Singh. Biotechnol Bioeng, 108, (2), 286-295, (2011).

3. K. Esau. Anatomy of Seed Plants. 2'ed. New York, (1977).

4. T.A. Morrison, H.G. Jung H. G., D.R. Buxton, R.D. Hatfield, Crop Sci., 38, 455-460, (1998).

5. P.J. Harris, R.D. Hartley, Nature, 259, 508-510, (1976).

6. A. Chesson, G.L. Provan, W. Russel, L. Scobbie. J. Sci. Food Agri., 73,10-16, (1997).

7. M. Corcel, M.F. Devaux, F. Guillon, C. Barron Comp. Electron. Agri., 127, 281-288, (2016). 